\title{
Distributed System Design for the Control and Evaluation of Engagement Simulations
}

\author{
Won K. Ham, Yongho Chung, and Sang C. Park, Member, IACSIT
}

\begin{abstract}
This paper introduces a system design methodology for controlling engagement simulations and evaluating the results of simulations. An engagement simulation system covers sophisticated operations of combat entities in a synthetic battlefield. Therefore, the system represents behaviors of combat entities to simulate a combat scenario and results of the simulation. However, there are factors which affect simulation execution, such as detection probability, moving speed, and moving path, and the result of a simulation can be distorted by unreliable values of those factors. For the useful simulation system of engagement, the system should be controllable by users for modifying inadequate values of important factors, and the results of simulations should be evaluated based on the change of effective factors. In this study, we define three types of effective factors and explain how to control and evaluate the defined factors.
\end{abstract}

Index Terms - Control, engagement, evaluation, simulation system.

\section{INTRODUCTION}

Recently, military modeling and simulation (M\&S) is important and inevitable in the planning and operation of national defense strategies and war fighting efforts. This causes from the complex concept of future warfare that are many weapon systems are mutually and complicatedly linked each other. Modern weapon systems have highly increased abilities by the development of the scientific technologies in comparison with weapons' history. The modern weapon systems are capable to process information from a battlefield to create intelligence for their operations, and the weapon systems share their intelligence to improve their tactical decisions. This concept of the modern warfare, such as network-centric warfare (NCW) concept (see Fig. 1), is not the equal to the simple concept of the past. Therefore, modeling these high technologic weapon systems and battle field is not a simple question to gain the answer from mathematical equations. $M \& S$ is necessary to achieve cost-effectiveness weapon systems development, to examine the synergism of various weapon systems, and experiment environmental effects of the battle field to weapon systems, except for traditional purposes, such as training [1].

$M \& S$ is a tool that is necessary and important in the acquisition of defense technologies and weapon systems, but it is still ambiguous to apply it into practice. Because a single weapon system is designed to perform various operations in

Manuscript received February 1, 2014; revised March 23, 2014.

The authors are with the Department of Industrial Engineering, Ajou University, Suwon, South Korea (e-mail: lunacy@ajou.ac.kr, yongho1230@gmail.com,scpark@ajou.ac.kr). the NCW concept, various information types and process functions for modeling each single weapon system are required. Therefore, the integration of technologies composing each weapon system should be represented in simulations, but the problem of the representation is that is impossibly difficult to represent them as traditional solutions, such as mathematical equations. For the representation of the modern warfare, a simulation system should be constructed using components for the involved functions and data with operations. In other words, a simulation is constructed based on assembly of componentized systems by a simulation objective. Distributed simulation system helps to achieve this modern M\&S objectives by integration of legacy simulators.

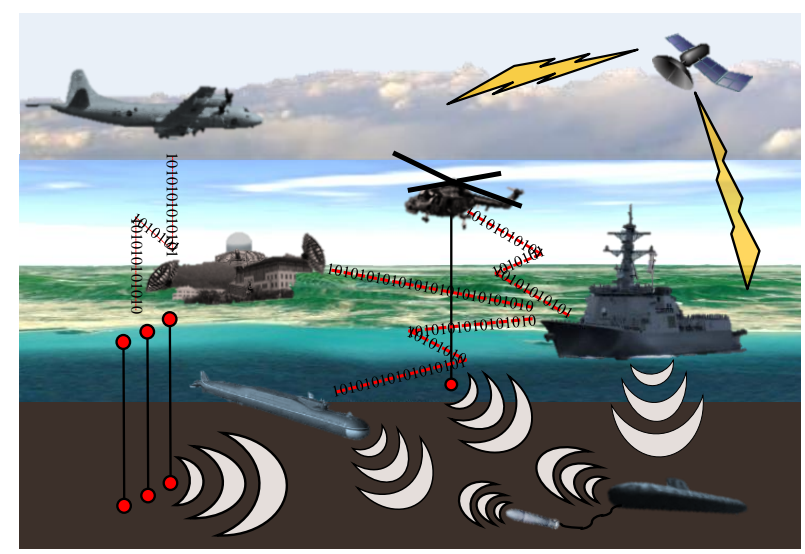

Fig. 1. NCW concept based operation.

Existing simulation systems have been confined to single, isolated developed for single purpose. However, to build new simulation system for modern warfare, which includes large-scaled and complex resources, require tremendous time, cost, and efforts. Thus, distributed simulation system is in demand to obtain systems for various purposes with combination a multitude of individual simulations of existing simulators into larger simulations [2]. US Department of Defense (DoD) founded Modeling and Simulation Coordination Office (M\&SCO, originally established as Defense Modeling and Simulation Office (DMSO)), and the M\&SCO has been leading DoD M\&S standardization and empowering $M \& S$ capabilities to support the full spectrum of military activities and operations. M\&SCO developed and released the IEEE 1516 High Level Architecture (HLA) standard which is distributed simulation architecture designed to facilitate interoperability and promote software reusability [3]. Run-Time Infrastructure (RTI) is implementation of HLA and fulfillment of objectives of distributed simulation system.

Simulation systems for the representation of engagements should have purposes that are the description of sophisticated behaviors by the functions and interactions of weapon 
systems during the short duration from minutes to hours. The expected outcomes of the engagement simulation model are such as survivability, vulnerability, and detection-ability of weapon systems [4]. In order to construct an engagement simulation system based on HLA/RTI, functions of weapon systems are componentized and connected with a HLA/RTI system and interactions among weapon systems are executed by data interchange in the system. However, monitoring, controlling, and evaluating an engagement simulation are hindered by distributed simulation information. For the evaluation of engagement simulation results, simulation factors should be monitored to trace the changes of values and events and be controlled to meet a simulation purpose during the execution of simulations. Therefore, distributed engagement simulation systems must include a component to monitor and control simulation factors for the evaluation of simulation results. The main objective of this paper is to propose a system design for the distributed engagement simulation that can be monitored, controlled, and evaluated. This paper explains the system design with an example that is the construction of a distributed underwater engagement system.

This paper is organized as follows. Section II explains the technical background of this paper, and Section III details the distributed system design of the engagement simulation system. Finally, Section IV summarizes the main conclusion of this paper.

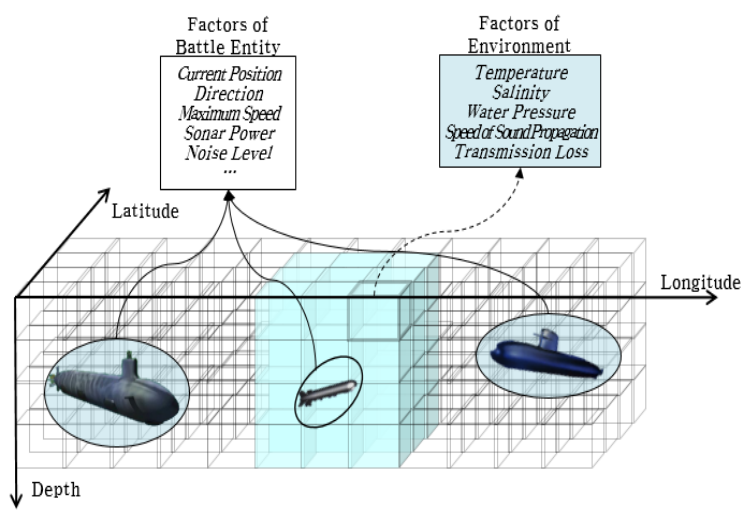

Fig. 2. Synthetic battlefield for Underwater engagement.

\section{TECHNICAL BACKGROUND}

\section{A. HLA/RTI Based Distributed System}

The HLA based distributed system is one of the indirect communication systems that follows P-S (Publish-Subscribe) paradigm and this is designated by a server and clients, for distributed event-based systems. In the system, clients are assumed as publishers and/or subscribers, publishers publish structured events to an event service and subscribers express interests on particular events through subscriptions [5].

In the simulation system that is based on HLA, a client is called as a federate, and federates publish/subscribe information to/from the RTI (Runtime Infra Structure) server. RTI is software that is an implementation of HLA. The whole simulation system is termed as federation. This specifies, in advance the start of a simulation, a set of federate applications and a common Federation Object Model (FOM). FOM is a specification that defines the information which is exchanged at runtime to achieve the given objectives of federation. It includes communication detail of federates, such as object/interaction classes which are ways of communication among federates. In order to communicate with the RTI server, a federate is indispensable to have an interface. This is referred to as Simulation Object Model (SOM), and SOM contains information on what its federate is going to publish and/or subscribe data of the classes that are defined in FOM.

In HLA based distributed system, the application connects RTI server as a federate that environment federate subscribes synthetic battlefield information and publishes the pertinent environmental data. The battle simulator federate publishes the synthetic battlefield information and it subscribes environmental data of the requested battlefield which is published by the environment federate. It uses the subscribed data to increase or decrease the characteristics of battle objects that are affected by the environmental effects. According to the variation, the results of an engagement simulation are changeable.

\section{B. Synthetic Battlefield for Engagement Simulations}

In HLA/RTI systems and any military M\&S applications, the environmental data are crucial in the depiction of the synthetic battle field situations [6]. The representation of the environment to the synthetic battlefield is a critical factor in the simulation for naval warfare. In order to identify the essential properties of the underwater battlefield, we require understanding the underwater warfare situation. In underwater engagement, sonar (an acronym for SOund Navigation And Ranging) is the mean that detects objects under the sea by using sound propagation. A submarine has several sonar systems along with the various purposes. For example, exploring the bathymetry, reconnaissance and detecting objects, identifying detected objects and tracing enemies, using passive and active sonar systems. A submarine force has high capability to conceal itself, and it usually sails deep underwater. The submarine force can detect the surface ship force much easier compared to that of the surface ship force which detects the submarine force. It is precisely difficult to detect a submarine force to surface the ship force by itself, due to the environmental characteristics of underwater regarding sound propagation efficiency. The main assault weapon is the torpedo. It also has a sonar system to navigate and detect the target [7].

By considering the previous description of the underwater warfare, the sonar is the most important system of the warfare. The performance of sonar operations is affected by variations in sound speed. However, the speed of sound in seawater is not a constant value, and it varies by temperature, salinity, and water pressure. Fig. 2 shows spatial information and required attributes for the synthetic battlefield of underwater engagement.

\section{SYSTEM DESIGN}

For the construction of a system for underwater engagement simulation, there are required three types of federates. Firstly, the simulator federate executes actual simulations of user-input scenarios. Secondly, the 
environment federate provides environmental data to construct the synthetic battlefield. Lastly, the sonar operation federates processes environmental data to calculate detection probabilities among simulation entities. This federation design is described in Fig. 3.

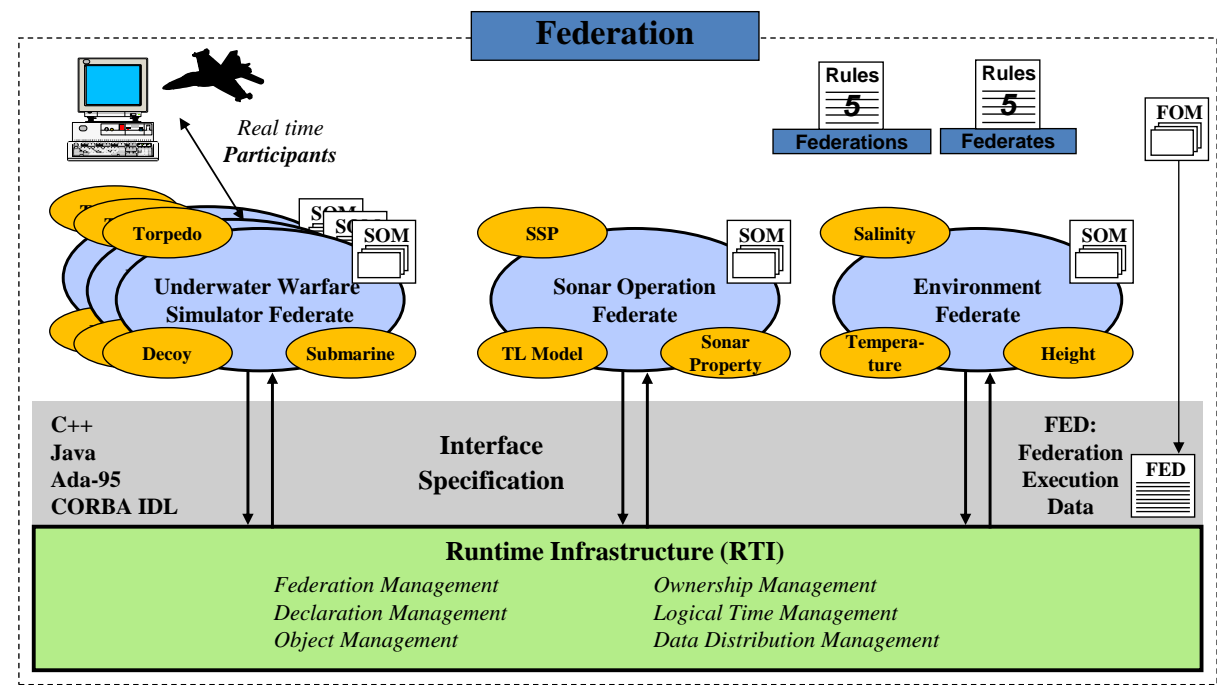

Fig. 3. Federation for underwater engagement.

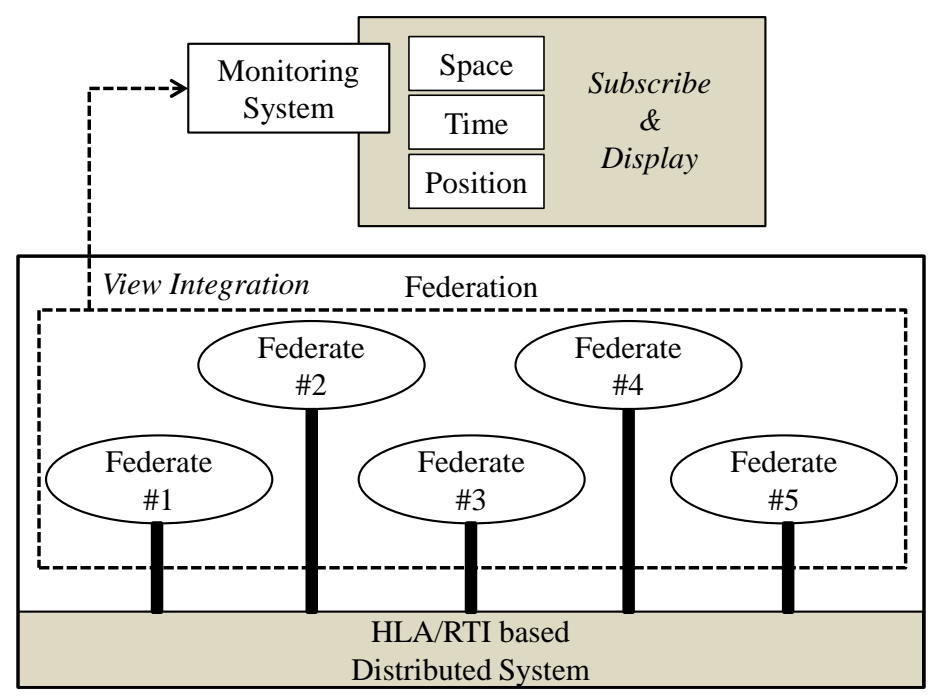

Fig. 4. Monitoring for federation

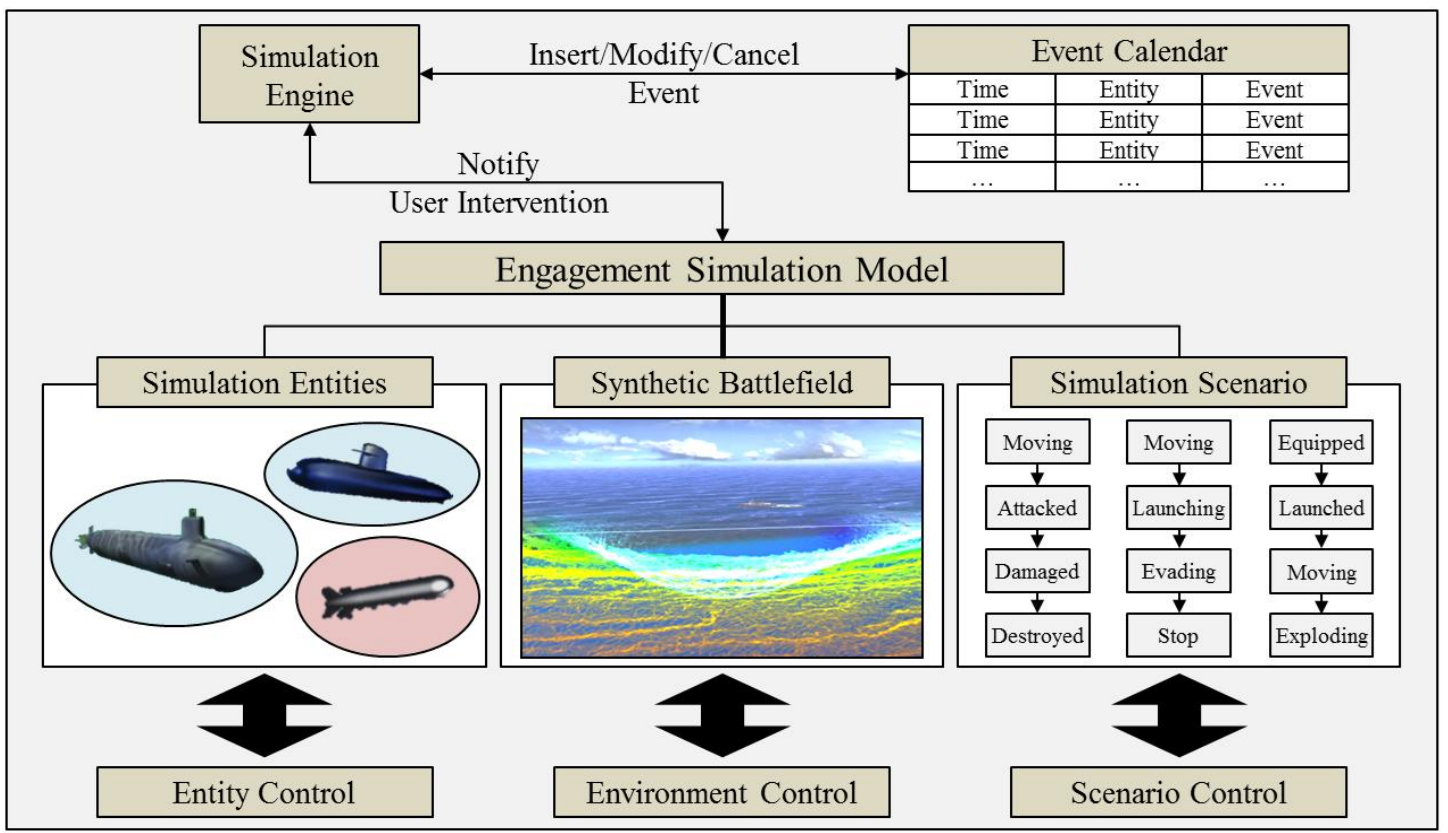

Fig. 5. Simulation control. 
The monitoring system of the HLA/RTI based simulation system is to integrate information flowing on the distributed federates of a federation. Derived factors to monitor the simulation system are related information about space, time, and position. Therefore, the system traces the positions of simulation entities by time lapse and the environment information of a space taken a place for engagement. The derived monitoring factors as following:

1) Simulation entities for weapon systems: maximum velocity, acceleration, rotate-ability, weapon equipment state (such as light/heavy torpedo, ASROC, harpoon missile, decoy, and jammer), detection ability (such as active sonar power, detection threshold, detectable angle, and noise emission), and position state (such as current latitude, longitude, depth, course, and speed)

2) Simulation entities for munitions: munitions type, parent entity, target entity, name, maximum velocity, acceleration, rotate-ability, maximum operable time, position state (such as current latitude, longitude, depth, course, and speed)

3) Synthetic battlefield information: temperature, salinity, water pressure, speed of sound propagation, transmission loss

The user of the simulation system traces and observes the change of those derived factors as represented in Fig. 4.

The initial configuration of a simulation system is set the system up by user, which is to meet the purpose of a simulation scenario. As the simulation is going, simulation factors are changed, and the user controls those factors to get appropriate simulation results. In order to control a simulation system in operation, this paper sorts three types of simulation system control as following (see Fig. 5).

1) Simulation entity: Controlling human intervention to control organization of simulation entities during a simulation by adding, modifying, and deleting a simulation entity during an execution of a simulation on the synthetic battlefield.

2) Synthetic battlefield: Controlling human intervention to control condition of the synthetic battlefield during a simulation by modifying data of the synthetic battlefield during an execution of a simulation.

3) Engagement scenario: Controlling human intervention to control behavior scenarios of simulation entities during a simulation by input an order to a simulation entity by a user during an execution of a simulation on the synthetic battlefield.

Therefore, the user of a simulation system is able to experiment 1) the efficiency of system operations and the synergy power of weapon systems by the simulation entity control, 2) the vulnerability and efficient operation procedures of weapon systems along the change of synthetic battlefield by the synthetic battlefield control, 3) the tactical ability and the various operation scenarios of weapon systems by the engagement simulation scenarios control.

After a simulation is finished, the result of the simulation is evaluated to assess weapon systems and operations. In order to evaluate simulations, the user of the simulation system looks the result of the change of monitored factors. Finally, the user can identify the efficiency of operations and the synergy, strength and vulnerability of weapon systems based on environmental conditions.

\section{SUMMARY AND FUTURE STUDY}

Defense $M \& S$ is a key to achieve several future warfare paradigms that are about minimum waste and maximum effects. By applying $M \& S$ for the development and deployment of weapon systems is efficient and it is essential to verify the objective, capacity, durability, effectiveness, productivity, and life cycle of weapon systems. M\&S allows experiments of the new weapon system, before investing on the resources. So, we can find out the problems and modify in advance. Consequently, $M \& S$ reduces costs, time, and efforts to develop and deploy weapon systems. M\&S for underwater warfare is devised for the evaluation of weapon systems, such as submarine, torpedo, decoy, and sonar systems, by the representation of engagements by using weapon system models on synthetic battlefield. In underwater warfare situations, the weapon systems are very dependent upon the environmental information of the battlefield. Therefore, interaction between the systems and battlefield must reflect on the simulation. In order to describe the interaction in HLA/RTI based distributed simulation system, environment federate is taken place to provide environmental data to the battle simulator.

The proposed framework of this paper was developed to integrate distributed information in HLA/RTI based simulation systems, and it allows users can control and evaluate simulation results. Thus, the results of simulations can facilitate meaningful insights in the development and deployment of weapon systems.

\section{ACKNOWLEDGMENT}

This work was supported in part by the Defense Acquisition Program Administration (DAPA), and Korea Association of Industry Academy and Research Institute (C00035790100384919) under the Contract No. UD110006MD (DAPA) \& UD120035JD (ADD). The authors wish to express sincere gratitude for the financial support.

\section{REFERENCES}

[1] S. C. Park, Y. Kwon, K. Seong, and J. J. Pyun, "Simulation framework for small scale engagement," Computers and Industrial Engineering, vol. 59, pp. 463-472, 2010.

[2] Australian Defense Simulation Office, Distributed Simulation Guide, Canberra, Australia: Department of Defence, 2004.

[3] M\&SCO. [Online]. http://www.msco.mil/descMSCO.html.

[4] P. A. Hawley and R. A. Blauwkamp, "Six-Degree-of-Freedom digital simulations for missile guidance, navigation, and control," Johns hopkins APL technical digest, vol. 29, no. 1, pp. 71-84, 2010.

[5] G. Coulouris, J. Dollimore, T. Kindberg, and G. Blair, Distributed systems: Concepts and design, fifth ed., USA: Addison-Wesley, 2012.

[6] P. Foley, F. Mamaghani, and P. Birkel. The SEDRIS Development Project. [Online]. Available: http://www.sedris.org

[7] P. H. Brady and D. McCormick, "Undersea warfare division: A message from the naval undersea warfare center," National defense industrial association, vol. 24, pp. 1-10, 2008. 


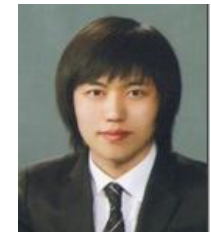

Won K. Ham has been received his bachelor degree in industrial and information system engineering and a master degree in industrial engineering, Ajou University, Korea. He is now a Ph.D candidate in industrial engineering, Ajou University, Korea, and he is a member of modeling and simulation laboratory, which is an affiliation of department of industrial engineering, Ajou University. $\mathrm{He}$ is interested in distributed simulation system, synthetic environment, and manufacturing simulation system.

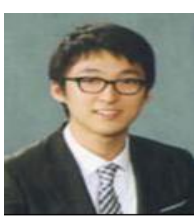

Yongho Chung has been received his bachelor degree in industrial and information system engineering and a master degree in industrial engineering, Ajou University, Korea. He is now a Ph.D candidate in industrial engineering, Ajou University, Korea, and he is a member of modeling and simulation laboratory, which is an affiliation of department of industrial engineering, Ajou University. He is interested in kinetic modeling, and mesh generation.

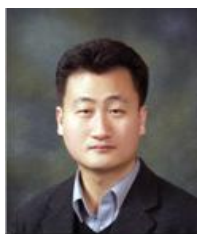

Sang C. Park was granted his bachelor in 1994. He received his master and Ph.D. degrees in industria engineering, Korea Advanced Institute of Science and Technology (KAIST). After his doctor's course, he had been a senior researcher of Cubictek, Korea, for 2 years from 2000. In 2002, he moved into DaimlerChrysler and took as role of research specialist, ITM Dept, for 3 years. Currently, he is an associate professor in Dept. of industrial and information systems engineering, Ajou University, Korea, since 2004. He is interested in modeling and simulation (M\&S), combat simulation for defense, digital manufacturing system, computer graphics and computational geometry and sculptured surface modeling and NC machining. 